\title{
Prognostic factors of renal outcomes after heart transplantation: a nationwide retrospective study
}

\author{
Junseok Jeon ${ }^{1}$, Hyejeong Park ${ }^{2}$, Youngha Kim², Danbee Kang ${ }^{2}$, Jung Eun Lee ${ }^{2}$, Wooseong Huh ${ }^{1}$, Yoon-Goo Kim', \\ Dae Joong Kim ${ }^{1}$, Juhee $\mathrm{Cho}^{2}$, Hye Ryoun Jang ${ }^{1}$ \\ 1Department of Internal Medicine-Nephrology, Samsung Medical Center, Seoul, Korea
${ }^{2}$ Department of Clinical Epidemiology, Samsung Medical Center, Seoul, Korea
}

Background: Renal dysfunction after heart transplantation (HT) is associated with poor survival. Predicting renal outcome after HT is a substantial but difficult issue. We investigate the predictive factors of renal outcomes after HT using nationwide cohort data.

Methods: In this retrospective cohort study using the Korean Intensive Care Unit National Data registry of the Health Insurance Review and Assessment database, 654 patients who received HT between 2008 and 2016 and survived until discharge after HT were analyzed.

Results: The median (interquartile range) age was 52 years (40-60 years), and 68.1\% were male. Perioperative renal replacement therapy (RRT) was administered to $27.8 \%$ of patients. During 2.8 years of median follow-up, end-stage kidney disease (ESKD) developed in 12 patients (1.8\%). In a fully adjusted model, RRT >3 weeks (hazard ratio [HR], 8.64; 95\% confidence interval [Cl], 3.17-23.51) and the use of inotropes/vasopressors ( $\mathrm{HR}, 6.98 ; 95 \% \mathrm{Cl}, 2.10-23.17)$ and angiotensin-converting enzyme inhibitor/ angiotensin receptor blocker $(\mathrm{HR}, 0.24 ; 95 \% \mathrm{Cl}, 0.08-20.71)$ were associated with ESKD. Pre-existing renal disease tended to be associated with ESKD (HR, 3.19; 95\% Cl, 0.87-11.71). Among the 561 patients without pre-existing renal disease, $104(18.5 \%)$ developed chronic kidney disease (CKD). Age (HR 1.03; 95\% Cl, 1.01-1.04), extracorporeal membrane oxygenation (HR, 1.53; 95\% $\mathrm{Cl}, 1.17-2.00$ ), and RRT (RRT 1-21 days: $\mathrm{HR}, 1.76 ; 95 \% \mathrm{Cl}, 1.28-2.41$ and $\mathrm{RRT}>21$ days: $\mathrm{HR}, 3.69 ; 95 \% \mathrm{Cl}, 1.41-9.68$ ) were associated with the development of CKD after HT.

Conclusions: Our nationwide cohort study demonstrated that perioperative RRT was a predictor of poor renal outcomes after HT. These results suggest that an active renoprotective strategy is required during the perioperative period.

Corresponding author: Hye Ryoun Jang

E-mail: shinehr@skku.edu

(c) The Korean Society for Transplantation

This is an Open Access article distributed under the terms of the Creative Commons Attribution Non-Commercial License (http://creativecommons.org/licenses/by-nc/4.0/) which permits unrestricted non-commercial use, distribution, and reproduction in any medium, provided the original work is properly cited. 